\title{
IDENTIFICACIÓN Y DESCRIPCIÓN DE SÍNTOMAS DE LOS PRINCIPALES PATÓGENOS ASOCIADOS AL CULTIVO DE MARAÑÓN (Anacardium occidentale L.) ORGÁNICO Y CONVENCIONAL, EN LEÓN, NICARAGUA
}

\section{IDENTIFICATION AND SYMPTOMS DESCRIPTION OF THE MAIN PATHOGENS ASSOCIATED TO CASHEW NUT (Anacardium occidentale L.) IN ORGANIC AND CONVENTIONAL FARMING IN LEON, NICARAGUA}

\section{Jiménez-Martinez Edgardo ${ }^{1}$ Gómez-Martinez Jorge ${ }^{2}$}

${ }^{1} \mathrm{PhD}$ en Entomología, Universidad Nacional Agraria, UNA, Profesor Titular, Docente-Investigador

${ }^{2} \mathrm{MSc}$. en agroecología y desarrollo sostenible, UNA, Profesor adjunto, Docente-Investigador

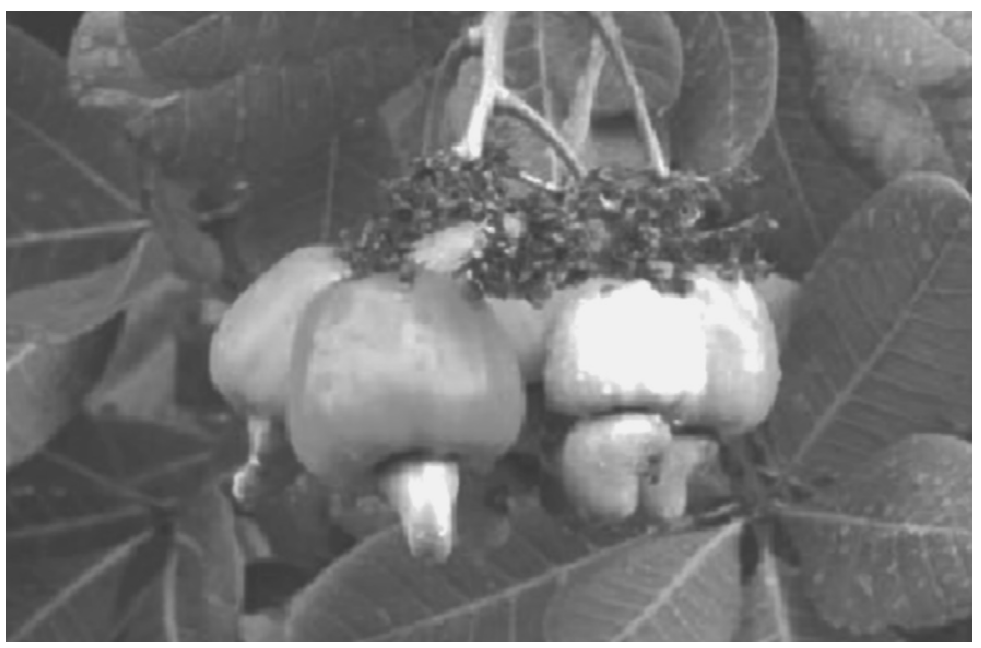

\section{RESUMEN}

El presente estudio se realizó con el objetivo de identificar y describir los principales agentes causales de daño asociados al cultivo de marañón orgánico y convencional. Este estudio se realizó durante el período Julio 2009 a Marzo del 2010, en la comarca Chacraseca, del departamento de León. Mediante muestreos sistemáticos se evaluó la incidencia y severidad de estos agentes en el dosel delos árboles, para ello se seleccionaron cinco sitios fijos de muestreos en parcelas orgánicas y convencionales. Se colectaron muestras de hojas con signos y síntomas de posibles enfermedades, las muestras se llevaron al laboratorio de micología de la Universidad Nacional Agraria para su respectivo análisis patológico. Las variables evaluadas fueron: incidencia y severidad de manchas necróticas (Pestalotia $s p$ ) por ramas con hojas, Incidencia y severidad de manchas anaranjadas (Cephaleuros $s p$ ) por ramas con hojas, se midió el área bajo la curva de progreso de la enfermedad (ABCPE) de manchas necróticas y manchas anaranjadas. Los resultados fueron los siguientes: se identificó y se describió los síntomas de Pestalotiasp, y Cephaleurossp, estos resultaron ser los principales agentes causales de daño foliar en marañón, en este mismo estudio, se encontró mayor porcentaje de incidencia y severidad de manchas necróticas y manchas anaranjadas en parcelas con manejo orgánico que en parcelas con manejo convencional. La menor ABCPE para manchas necróticas se presentó en parcelas con manejo orgánico, $\mathrm{y}$ la menor $\mathrm{ABCPE}$ para manchas anaranjadas se presentó en parcelas con manejo convencional.

\begin{abstract}
This study was conducted with the objective to identify and to describe the major causative damage agents associated to cultivated organic and conventional cashew nut. The study was conducted during the period from July 2009 to March 2010, in the community of Chacraseca in the department of León. Using systematic sampling it was assessed the incidence and severity of these damaging agents in the area of the canopy of trees; five fixed sampling sites in organic and conventional plots were selected. Leaves with signs and symptoms of possible diseases were collected; leaves samples were brought to the laboratory of Mycology of the National Agrarian University for their respective pathologic analysis. Evaluated variables were as follow: Incidence and severity of necrotic spots (Pestalotiasp) by branches with leaves, incidence, and severity of orange spots (Cephaleurossp) by branches with leaves, the area under the disease progress curve (ABCPE) of necrotic spots and orange spots were measured. Results were as follow: It was identified that Pestalotiasp, and Cephaleurossp, were the main causative agents of foliar damage in cashew nut; in this same study, it was found a greater percentage of incidences and severity of necrotic spots and orange spots in plots with organic management than in plots with conventional management. The lower ABCPE for necrotic spots are presented in plots with organic management, and the lesser $\mathrm{ABCPE}$ for orange spots are presented in plots with conventional management.
\end{abstract}


$\mathrm{L}$ as enfermedades de las plantas son de gran importancia debido a que pueden debilitar la salud de la planta de forma directa, afectando la producción, utilización, calidad y vida de almacenaje de las frutas (Castaño, 1994). A nivel mundial las enfermedades más importantes de las plantas son aquellas que proporcionan el mayor porcentaje de alimento a la humanidad, en países desarrollados, en donde los alimentos son abundantes, las enfermedades de las plantas son importantes porque causan pérdidas económicas a los productores, incrementan el uso de plaguicidas, aumento en los precios de los productos al consumidor y la destrucción del medio ambiente (Castaño, 1994).

El marañón como cualquier otro cultivo, es afectado por diversas enfermedades, en países como Brasil y El Salvador se reportan enfermedades tales como: el moho prieto, gomosis (Lasidoplodia theobromae Pat.), necrosis y momificación (Macrophoma $s p$ ), mancha angular y la mancha producida por algas (Galdanez, 2004).

El CIPRES, 2008, reporta que en las plantaciones de marañón del occidente de Nicaragua (León y Chinandega), se han reportado dos enfermedades causadas por hongos. Estas enfermedades son: Antracnosis (Colletotrichum $s p$ ) y el Mildiu polvoriento (Oidium $s p$ ), sin embargo, se cree que existen otros patógenos que podrían estar afectando las plantaciones de marañón, las cuales en un futuro podrían convertirse en graves problemas para los productores de estas zonas.

Debido a la importancia económica de este cultivo para el occidente del país y debido al poco conocimiento que existe sobre aspectos fitosanitarios y de diagnóstico de patógenos asociados al marañón, se realizó este estudio con el objetivo de identificar, describir y calcular la incidencia y severidad del daño de los principales patógenos asociados al cultivo del marañón.

\section{MATERIALES Y MÉTODOS}

Localización de la zona de estudio. El estudio se llevó a cabo entre los meses de julio del 2009 a marzo 2010 en la comarca Chacraseca ubicada a $5 \mathrm{~km}$ al sureste de la cabecera departamental (León) en las coordenadas 12 26', 58' latitud norte y $86^{\circ} 53^{\prime}, 52^{\prime \prime}$ longitud oeste, con altura de $109.21 \mathrm{msnm}$ (INETER, 2009).

Ubicación y descripción de las parcelas en estudio. El estudio se realizó en la comarca Chacraseca, departamento de León. Para la realización de este estudio, se seleccionaron parcelas ya establecidas en seis fincas tres de ellas manejadas por los productores de manera orgánica y tres de manera convencional, las fincas orgánicas son propiedad de los productores Agustín Reyes, Reymundo Mendoza y Meláneo Acosta, las fincas convencionales son de los productores de Orfília Reyes, Gustavo Munguía y William Gurdián.
En cada finca se seleccionaron 5 sitios fijos distribuidos en cada parcela. Cada sitio consistió en 2 árboles de marañón (10 árboles por finca) y en cada árbol se seleccionaron3 ramascomo unidades de muestreo para un total de 30 ramas por finca ( 5 sitios x 2 árboles x 3ramas) las ramas fueron se seleccionaron como unidades fijas de muestreo, el muestreo fue estratificado iniciando de la parte superior a la parte inferior del árbol.

Condiciones climatológicas registradas durante los meses de muestreo, febrero 2010 a mayo 2010 en León. Los datos climatológicos fueron obtenidos del Instituto Nicaragüense de Estudios Territoriales (INETER). Las temperaturas se mantuvieron entre un rango de $26-28{ }^{\circ} \mathrm{C}$, registrándose las más bajas entre los meses de octubre a enero y en el período de febrero a marzo se registraron las mayores temperaturas con 28.4 y $28.7{ }^{\circ} \mathrm{C}$. Las precipitaciones más altas se registraron entre los meses de Febrero y Marzo del 2010 con un promedio de 35.6 milímetros para ambos meses. La humedad relativa varió entre un rango de 80-90 \% durante el periodo de estudio (agosto 2009 y marzo del 2010).

Variables evaluadas en el estudio. Incidencia de Manchas necróticas (Pestalotia $s p$ ) por ramas con hojas, incidencia de Manchas anaranjadas (Cephaleuros $s p$ ) por ramas con hojas, severidad de Manchas necróticas (Pestalotia sp) por ramas con hojas, severidad de Manchas anaranjadas (Cephaleuros $s p$ ) por ramas con hojas, área bajo la curva de progreso de la enfermedad (ABCPE) de manchas necróticas y área bajo la curva de progreso de la enfermedad (ABCPE) de manchas anaranjadas.

Metodología de muestreo de enfermedades en el campo. Se realizaron muestreos semanales de incidencia y severidad de daño a partir del 1 de julio del año 2009 hasta el 5 de marzo del 2010 obteniéndose un total de 36 toma de datos. Para realizar estos muestreos, se seleccionaron tres parcelas con manejo orgánico y tres parcelas con manejo convencional de 0.7 ha cada una, en cada parcela se seleccionaran cinco sitios fijos de muestreo, cada sitio estaba compuesto por dos árboles entre los cinco y siete años de edad, cada árbol se dividió en tres estratos (parte superior, media e inferior), en cada estrato se seleccionaba una rama lateral con todas sus hojas para obtener la incidencia y severidad de daño por rama. Para el caso de la aplicación de la escala de severidad de daño, el total de hojas de cada rama, era considerado como un cien por ciento.

\section{Evaluación de enfermedades}

Incidencia. Para determinar el porcentaje de incidencia de enfermedades se utilizó la siguiente fórmula:

Incidencia $(\%)=\frac{\text { Total de hojas con síntomas }}{\text { Total de hojas muestreados }} \times 100$ 
Severidad. Con el fin de facilitar la realización de este estudio, se propusieron tres escalas (tabla 1 y 2) para medir el nivel de daño de las diferentes enfermedades foliares en marañón. Estas escalas consistieron en la estimación visual del porcentaje afectado del área foliar (hojas) con respecto al total del follaje de la rama.

Tabla 1. Escala de severidad para mancha anaranjada (Cephaleuros $s p)$. Propuesta por Jiménez-Martínez y Gómez 2009

\begin{tabular}{|c|c|}
\hline Grado & Descripción \\
\hline 0 & $0 \%$ de la rama afectada \\
\hline 1 & $\begin{array}{l}1-10 \% \text { de todas las hojas afectadas con síntomas de } \\
\text { manchas anaranjadas }\end{array}$ \\
\hline 2 & $\begin{array}{l}11-20 \% \text { de todas las hojas afectadas con síntomas de } \\
\text { manchas anaranjadas }\end{array}$ \\
\hline 3 & $\begin{array}{l}21-30 \% \text { de todas las hojas afectadas con síntomas de } \\
\text { manchas anaranjadas }\end{array}$ \\
\hline 4 & $\begin{array}{l}31-40 \% \text { de todas las hojas afectadas con síntomas de } \\
\text { manchas anaranjadas }\end{array}$ \\
\hline 5 & $\begin{array}{l}50 \% \text { de todas las hojas afectadas con síntomas de } \\
\text { manchas anaranjadas }\end{array}$ \\
\hline
\end{tabular}

Tabla 2. Escala de severidad para manchas necróticas (Pestalotia $s p$ ) en el cultivo de marañón.

Propuesta por Jiménez-Martínez y Gómez 2009

\begin{tabular}{ll}
\hline Grado & \multicolumn{1}{c}{ Descripción } \\
\hline 0 & $0 \%$ de afectación en hojas \\
1 & $1-10 \%$ de todas las hojas afectadas con síntomas de \\
manchas irregulares necrosadas \\
$11-20 \%$ de todas las hojas afectadas con síntomas de \\
manchas irregulares necrosadas \\
21- 30\% de todas las hojas afectadas con síntomas de \\
manchas irregulares necrosadas \\
31-40\% de todas las hojas afectadas con síntomas de \\
manchas irregulares necrosadas \\
41-100\% de todas las hojas afectadas con síntomas de \\
manchas irregulares necrosadas
\end{tabular}

Para obtener el grado porcentual de severidad se utilizó la fórmula general planteada por Vanderplank, 1963.

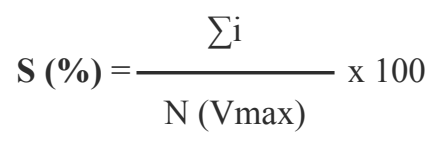

Dónde:

$$
\begin{aligned}
& \mathrm{S}=\text { Porcentaje de severidad. } \\
& \sum \mathrm{i}=\text { Sumatoria de valores observados. } \\
& \mathrm{N}=\text { Número de plantas muestreadas. } \\
& \text { Vmax = Valor máximo de la escala. }
\end{aligned}
$$

Área Bajo la Curva de Progreso de la Enfermedad (ABCPE). Con los registros de severidad por finca se calculó el área bajo la curva de progreso de la enfermedad (ABCPE) para manchas necróticas y manchas anaranjadas. La fórmula utilizada fue la propuesta por Cambell y Madden, (1990).

$$
\mathrm{ABCPE}=\sum\left[\begin{array}{ll}
x_{i+1}+x_{i}\left(t_{i+1}-t_{i}\right) & 2
\end{array}\right]
$$

Donde:

$x_{i}=$ Porcentaje de tejido afectado

$i=1$

$t=\underline{\text { Tiempo }}($ días $)$

$n=$ número de evaluaciones

Los datos de ABCPE obtenidos de cada finca fueron sometidos a un análisis de varianza para ver si esta variable fue igual o diferente en cada sitio.

Colección y procesamiento de muestras de hojas e identificación de enfermedades a nivel de laboratorio

Colección de muestras de hojas en el campo. Se recolectaron muestras de hojas con signos o síntomas de enfermedades, para la colecta de las hojas en el campo, se utilizaron tijeras, bolsas plásticas y papel toalla, el transporte de las muestras al laboratorio se hizo en hieleras portátiles, cada muestra se rotuló con fecha, sitio y número de parcela. Las muestras fueron procesadas en el laboratorio de micología de la Universidad Nacional Agraria en el km. 12 1/2 carretera norte, Managua, Nicaragua.

Análisis patológico de material vegetativo. Para realizar el análisis patológico del material vegetativo, se utilizaron técnicas de inducción de crecimiento de hongos, a partir de tejido vegetal enfermo posteriormente se sembró en medios de cultivos como: Agar-Agua (AA) y Papa Dextrosa Agar (PDA para inducir la esporulación de estructuras reproductivas del patógeno.

Cámara húmeda. Las muestras de tejido vegetal enfermo (hojas) se colocaron en platos petri o cajas plásticas con papel filtro, humedecido con agua destilada estéril para inducir a la esporulación de estructuras fructíferas de los hongos y su posterior identificación mediante el uso de microscopio.

Siembra de trozos de hojas con estructuras fructíferas y tejido infectado en AA y PDA. Primeramente se tomaron hojas con síntomas, posteriormente se realizaron pequeños cortes en la hoja de manera que solo quedara en la lesión la estructura como tal, luego se dejó reposar en agua destilada 
estéril por un minuto, se secó con papel filtro y se dejó reposar por 30 segundos, finalmente se sembró en platos petri con Agar-Agua (AA). El objetivo de este procedimiento es el de inducir el desarrollo y crecimiento de estructuras de reproducción, tanto de fase sexual (esclerocios, peritecios, apotecios, etc.) como asexual (cleistotecios, picnidios, acérvulos).Para la siembra de trozos de hojas con tejido infectado en medio de cultivo papa dextrosa agar (PDA), las muestras fueron primeramente desinfectadas en alcohol al $95 \%$ por 1 minuto, posteriormente se sembraron en platos Petri con PDA; estos platos petri se rotularon de la siguiente manera: nombre del cultivo, fecha de muestreo y nombre de la parcela. Finalmente, los platos se preservaron a temperaturas de 25-30 grados centígrados. Estos platos se revisaban diariamente con el propósito de observar estructuras reproductivas.

Identificación de hongos. Los géneros de hongos encontrados fueron identificados utilizando claves taxonómicas propuestas por Schaad, 1990. Las características morfológicas o de crecimiento como: color, forma de crecimiento, elevación de micelio y estructuras fructíferas se observaron en microscopio. También se usaron algunas referencias bibliográficas de autores como Monterrosa, 1996.

Análisis estadísticos. A partir de los datos obtenidos de incidencia y severidad se realizaron curvas del comportamiento de los principales patógenos causales a través del tiempo.

Para comparar la severidad de losagentes causales, se estimó el área bajo la curva de progreso de cada patógeno de cada sistema de finca, posteriormente a este parámetro se le realizó un análisis de varianza y separación de medias mediante la prueba de Tukey. Además, los datos de severidad obtenidos en el experimento fueron analizados mediante análisis de varianza de medidas repetidas (parcelas divididas en el tiempo) considerando las fincasde cada productor como la parcela grande y los tratamientos (sistemas de cultivos) como la parcela pequeña, posteriormente se hizo separación de medias mediante la prueba de Tukey tanto para la variable finca como para la variable sistema de cultivo, cuando estas resultaron significativas según el ANDEVA.

\section{RESULTADOS Y DISCUSIÓN}

Identificación de los principales patógenos. La identificación es el primer paso para la elaboración de estrategias de manejo en cualquier sistema de cultivo. Los principales agentes fungosos identificados en este estudio fueron, unas manchas irregulares necrosadas, ocasionadas por los géneros Pestalotia sp. y Cladosporium sp, Cabe mencionar que en los análisis patológicos de las muestras procesadas en el laboratorio, predominó el crecimiento por el género Pestalotia en medio de cultivo PDA, es por ello que en este estudio hacemos más énfasis en la incidencia de este género, dado que se considera el principal agente causal. También es importante aclarar, que en los análisis de laboratorio, ambos tipos de géneros se encontraron asociados causando los mismos síntomas en este cultivo. Otro de los agentes identificados fueron unas manchas anaranjadas causadas por el género Cephaleuros (según la MSc. Yaneth Gutiérrez Gaitán fitopatología y micóloga de la UNA, este agente es considerado como un tipo de alga que sobrevive y se reproduce en hojas de marañón) (comunicación personal). Es importante mencionar que los dos géneros de hongos identificados en este estudio (Pestalotia y Cephaleuros) son considerados parásitos débiles, los cuales aprovechan los daños primarios causados por otros agentes causales para poder invadir los tejidos de las plantas.

Comparación del porcentaje de incidencia de la mancha necrótica (Pestalotiasp.). Se comparó y se describe la incidencia de manchas necróticas causadas por el género Pestalotiasp en fincas de marañón con manejo orgánico y convencional en la comarca Chacraseca, León en el período comprendido julio 2009 a marzo 2010 (figura 1). De manera general se observa que la incidencia de este agente causal reflejó un comportamiento diferente para ambos sistemas de cultivo. En las fincas con manejo orgánico, la incidencia se presentó en todos los meses de muestreo, sin embargo, los mayores porcentajes de incidencia se presentaron en los meses de octubre y noviembre del 2009 con porcentajes de 88 y $90 \%$ respectivamente, posteriormente los porcentajes de incidencia fueron descendiendo de manera irregular hasta la última fecha de muestreo (marzo 2010). En las fincas convencionales la incidencia se incrementó de manera ascendente a partir del primer mes de muestreo (julio 2009) hasta el últimomes de muestreo marzo 2010) con $89 \%$ de incidencia. Al realizar el análisis de varianza y separación de medias para comparar los promedios de incidencias de manchas necróticas causadas por el género Pestalotiasp entre ambos sistemas de fincas, se encontró diferencias significativas $(P=0.0003)$, la mayor incidencia se presentó en las fincas con manejo orgánico con porcentajes promedios de $75.1 \%$, comparado con las fincas con manejo convencional que presentó porcentajes promedios de $60.7 \%$.

El síntoma inicial que se observó en el campo consistió en una necrosis parcial en las hojas de mayor edad. Los síntomas asociados a los daños por Pestalotia $s p$ fueron manchas foliares irregulares necrosadas con tejido deshidratado, manchas redondas necróticas en los bordes y peciolos tanto en el haz como en el envés de las hojas (Foto 1). Barnet y Hunter (1988) reportan estos hongos como oportunistas ya que atacan árboles débiles, o con lesiones y heridas, condiciones desfavorables o que ya han sido atacados por otros hongos primarios o daño físico.

El género Pestalotia, es un parásito oportunista, 
que coloniza tejido afectado, sin embargo, según Barnett y Hunter (1988) su presencia puede estar asociada a ataques de insectos chupadores. Este género se caracteriza principalmente por atacar el tejido joven de la planta y en condiciones de debilidad del hospedante las hojas presentan síntomas generalizados de marchitez.

Muñoz et al., (2007), describe que la supervivencia de este hongo queda asegurada en los restos de material vegetal enfermo o muerto por otras causas. En condiciones favorables el hongo forma los acérvulos que emiten masas de conidios infectivos que son transportados por lluvias, estos conidioforos son capaces de penetrar a través de heridas en el tejido debilitado o joven, donde estos se reproducen con mucha facilidad.

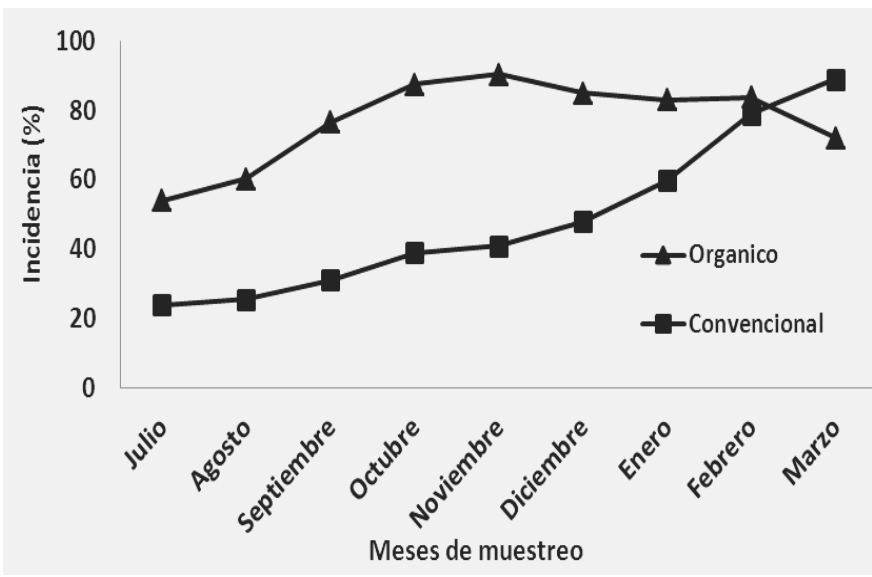

Figura 1. Porcentaje de incidencia de manchas necróticas (Pestalotia sp.) en el cultivo de marañón orgánico y convencional, durante el muestreo realizado entre los meses julio 2009 a febrero 2010 Chacraseca, León.

Comparación del porcentaje de incidencia de mancha anaranjada (Cephaleuros sp.). Se comparó y se describe la incidencia de manchas anaranjadas causadas por el género Cephaleuros sp en fincas de marañón con manejo orgánico y convencional en la comarca Chacraseca, León en el período comprendido julio 2009 a marzo 2010 (figura 2), en esta figura se observa que los porcentajes de incidencia reflejaron un comportamiento diferente en ambos sistemas de cultivo. En el sistema orgánico la incidencia de daño se presentó de manera ascendente, no obstante los mayores porcentajes se presentaron en los últimos meses de muestreo (febrero y marzo del 2010) con porcentajes de 46 y $53 \%$ respectivamente. En las fincas con manejo convencional la incidencia fue mayor en comparación con el sistema orgánico, sin embargo, los mayores porcentajes de incidencia se presentaron en los meses de septiembre y octubre del 2009 con porcentajes de 52 y $50 \%$. En el análisis de varianza y separación de medias realizado, se encontró diferencias significativas entre ambos sistemas de fincas $(P$
$=0.0001$ ), la mayor incidencia lo presentaron las fincas con manejo convencional con porcentajes promedios de $41.9 \%$, comparado con las fincas con manejo orgánico que presentó porcentajes promedios de $17.8 \%$.

Los síntomas iniciales de la mancha anaranjada que se observaron en el campo consistieron en pequeños puntos los que luego se combinaron y formaron puntos irregulares más grandes. Los daños asociados a estos síntomas encontrados en el campo fueron manchas redondas anaranjadas, las cuales se volvían grisáceas cuando alcanzaban un estado más avanzado (Foto 2). Algunos sitios de muestreo presentaron alta densidad poblacional, por lo cual los árboles se encontraban separados a poca distancia entre sí; permitiendo que el viento provocara heridas, y defoliación, una alta competencia por nutrientes, luz y espacio entre los árboles producto del roce entre estos, esto concuerda con lo citado por Sánchez (1988) quien reporta que los ambientes húmedos dentro de la copa de los árboles es propicio para el desarrollo y el establecimiento de este agente causal.

Según Ploestz (1998) la mancha anaranjada es muy frecuente en cultivos de la familia anacardiaceae y en muchos otros cultivos importantes de climas tropicales. Este patógeno es muy importante ya que puede reducir la fotosíntesis normal y la vitalidad del árbol; el agente se puede reconocer fácilmente por la presencia visible de puntos color naranja, el tamaño de los puntos varía entre 5 a 8 milímetros de diámetro en ambas superficies de la hoja.

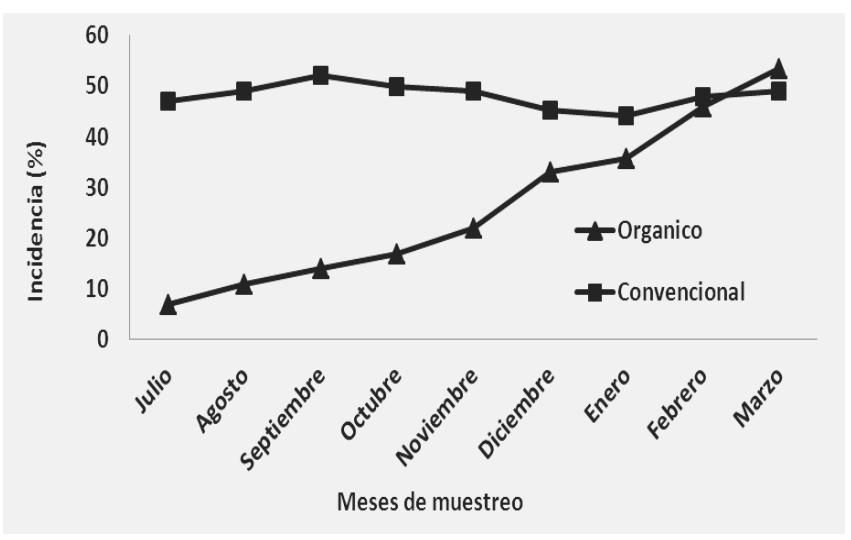

Figura 2. Porcentaje de incidencia de mancha anaranjada (Cephaleurossp) en el cultivo de marañón orgánico y convencional, durante el muestreo realizado entre los meses Julio 2009 a Marzo 2010 Chacraseca, León. 


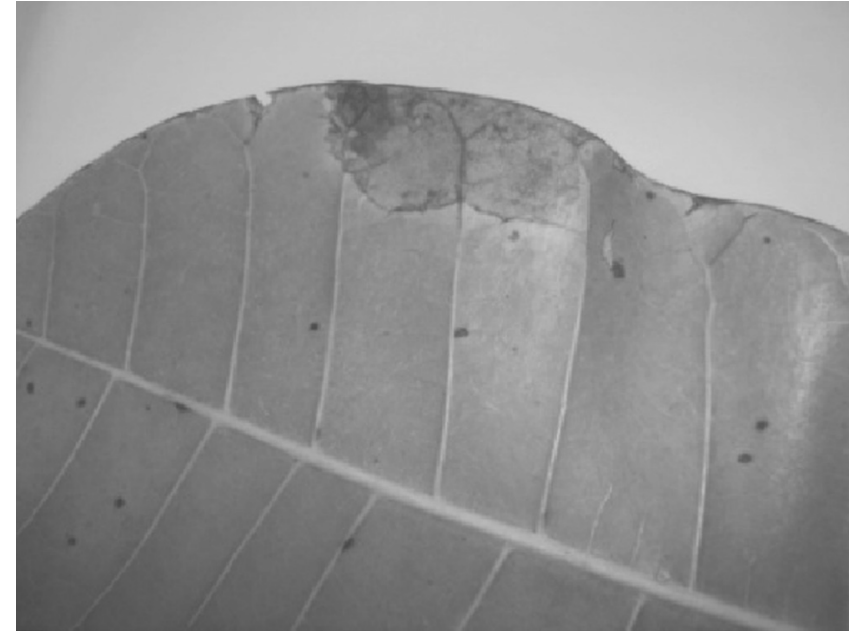

Foto 1 Manchas irregulares necrosadas. Pestalotia $s p$ ). Tejido deshidratado. Foto: Jorge Gómez, Identificación: Yannett Gutiérrez (Laboratorio micología, UNA).

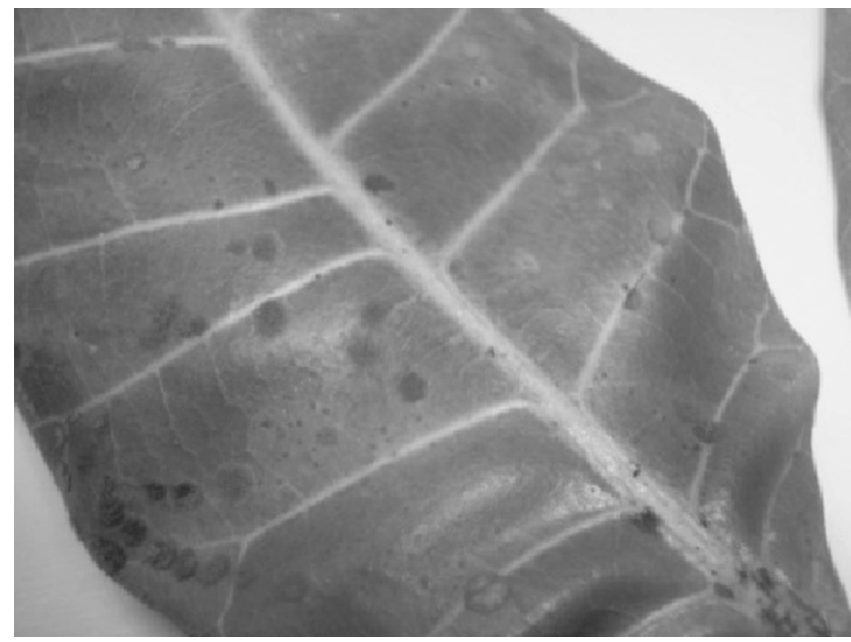

Foto 2. Mancha foliares por alga (Cephaleurossp). Foto: Jorge Gómez, Identificación: Yannett Gutiérrez (Laboratoriomicología UNA).

Comparación del porcentaje de severidad de mancha necrótica (Pestalotia sp.). Se comparó y se describe la severidad de manchas necróticas causadas por los géneros Pestalotia $s p$ en fincas de maranón con manejo orgánico y convencional en la comarca Chacraseca, León en el período comprendido julio 2009 a marzo 2010 (figura 3).Se observó que los porcentajes de severidad de manchas necróticas reflejaron un comportamiento de forma similar para ambos sistemas de fincas excepto en los últimos meses de muestreo. En las fincas con manejo orgánico la severidad se presentó en todos los meses de muestreo, pero los mayores porcentajes de incidencia se presentaron en los últimos meses de muestreo (febrero y marzo) del 2010 con 67 y $74 \%$. En las fincas con manejo convencional los mayores porcentajes de severidad se presentaron igualmente éntrelos meses de diciembre y enero con porcentajes de 48 y $45 \%$ de severidad respectivamente. Al realizar el análisis de varianza para comparar los promedios de severidad de mancha necrótica entre ambos sistemas de fincas, no se encontró diferencia significativa.

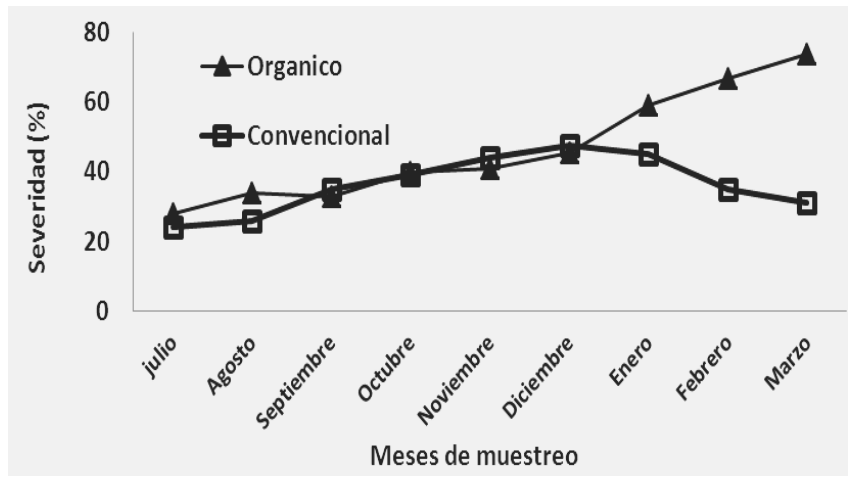

Figura 3. Porcentaje de severidad de manchas necróticas (Pestalotiasp) en el cultivo de marañón orgánico y convencional, julio 2009 a marzo 2010, Chacraseca, León.

Comparación del porcentaje de severidad de manchas anaranjadas (Cephaleuros sp.). Se comparó y se describe la severidad de manchas anaranjadas causadas por el género Cephaleuros $s p$ en fincas de marañón con manejo orgánico y convencional en la comarca Chacraseca, León en el período comprendido julio 2009 a marzo 2010 (figura 4). De manera general se observó que el género Cephaleuro sp presentó los mayores porcentajes de severidad en las fincas con manejo orgánico en comparación con las fincas con manejo convencional.

En las fincas con manejo orgánico la severidad se presentó en todas las fechas de muestreo, sin embargo, los mayores porcentajes se presentaron en los meses de agosto, septiembre y noviembre con 90 y $87 \%$ respectivamente, posteriormente los porcentajes fueron descendiendo de manera irregular hasta el último mes de muestreo (marzo del 2010 con $72 \%$ de severidad).

En las fincas con manejo convencional la severidad los mayores porcentajes de severidad se presentaron de forma ascendente a partir del primer mes de muestreo hasta alcanzar la mayor severidad en el último mes (marzo de 2010 con 53\%.Al realizar el análisis de varianza y separación de medias para la severidad de mancha anaranjada causada por Cephaleuros, se encontró diferencias significativas en ambos sistemas de fincas $(P=0.0001)$, los mayores porcentajes de severidad se encontraron en las fincas con manejo orgánico con porcentajes promedios de $7.19 \%$, comparado con las fincas con manejo convencional que presentó porcentajes promedios de $2.33 \%$ 


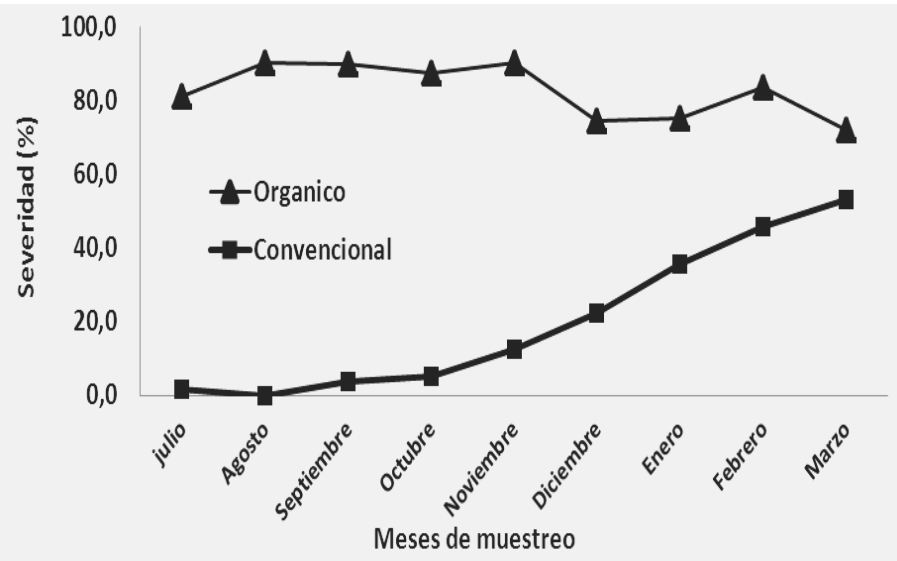

Figura 4. Porcentaje de severidad de mancha anaranjada (Cephaleuros $s p$ ) en el cultivo de marañón orgánico y convencional, durante el muestreo realizado entre los meses julio 2009 a marzo 2010 Chacraseca, León.

Comparación del Área bajo de la curva de progreso de la enfermedad (ABCPE) de manchas necróticas. Según el ABCPE total, realizado para manchas necróticas (Pestalotia $s p$ ), la mayor área bajo la curva se presentó en las fincas con manejo orgánico. En cambio la menor área bajo la curva se presentó en las fincas con manejo convencional, esto fue debido a que en este sistema los porcentajes de enfermedad fueron mayores. El mayor porcentaje-día se observó en las fincas con manejo convencional entre los meses de octubre y diciembre del 2009 con 730 y $540 \%$ por día (figura 5). Al contrario del análisis de varianza y separación de medias realizado para la severidad de manchas necróticas, el análisis de varianza y separación de medias para el área bajo la curva de progreso de la enfermedad, indica que existen diferencias significativas entre ambos sistemas de fincas $(P=0.0105)$. En general, los valores promedios más altos del área bajo la curva de progreso de la enfermedad, fueron encontrados en las parcelas con manejo orgánico con promedios de 43.7 y el sistema convencional con 40.9. Con estos resultados podemos afirmar que el sistema de manejo de las fincas orgánicas tuvieron algún efecto sobre el desarrollo de la enfermedad, pero también es posible que las condiciones ambientales que se presentaron en estos meses las cuales fueron humedades relativas medias $(77.9 \%)$ y temperaturas altas $\left(36{ }^{\circ} \mathrm{C}\right)$ favorecieron el desarrollo de la enfermedad.

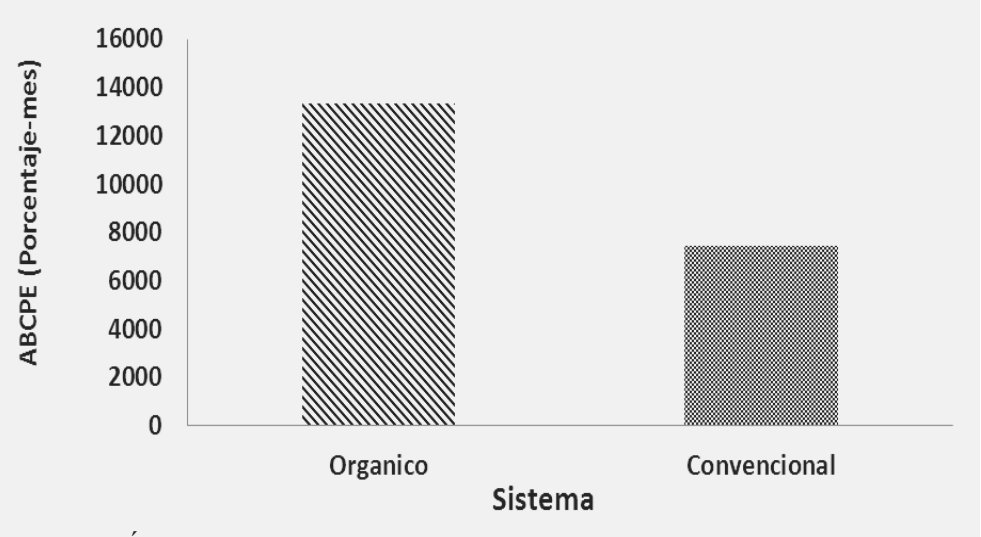

Figura 5. Área bajo la curva de progreso de la enfermedad (ABCPE) de manchas necróticas (Pestalotia $s p$ ) en fincas de marañón con manejo orgánico y convencional, entre los meses julio 2009 a marzo 2010 Chacraseca, León.

Comparación del Área bajo la curva de progreso de la enfermedad de manchas anaranjadas. El área bajo la curva de progreso de la enfermedad realizado para manchas anaranjadas, indica que el menor área bajo la curva se presentó en las fincas con manejo convencional. En cambio el mayor porcentaje-mes se observó en las fincas con manejo orgánico con $1750 \%$ por mes (figura 6).Al igual que el análisis de varianza y separación de medias para severidad de manchas anaranjadas, el análisis de varianza y separación de medias realizado para el área bajo la curva de progreso de la enfermedad indica que existen diferencias significativas entre los sistemas de fincas $(P=0.002)$, los mayores porcentajes de severidad se presentaron en las fincas con manejo orgánico con porcentajes promedios de $31.4 \%$, comparado con las fincas con manejo convencional que presentó porcentajes promedios de $17.2 \%$. Las características de los sistemas orgánicos como: abundancia de sombra y poca aireación en árboles fue propicio para el desarrollo y establecimiento de este agente causal.

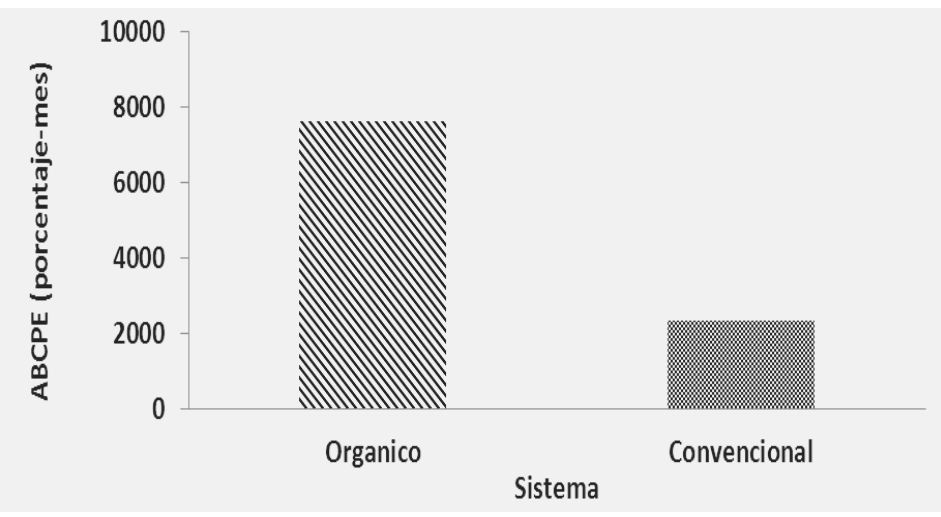

Figura 6. Área bajo de la curva de progreso (ABCPE) de manchas anaranjadas (Cephaleuros $s p$ ) en fincas de marañón con manejo orgánico y convencional, durante el muestreo realizado entre los meses julio 2009 a marzo 2010 Chacraseca, León. 


\section{CONCLUSIONES}

Los patógenos. Pestalotia $s p$, y Cephaleuros $s p$ ) fueron los principales patógenos asociados a manchas foliares en marañón.

La incidencia de Manchas necróticas (Pestalotia $s p$ ), y Manchas anaranjadas (Cephaleuros $s p$ ) fue mayor en fincas de marañón con manejo orgánico que en las fincas con manejo convencional.

La severidad de manchas necróticas (Pestalotia $s p$ ), y manchas anaranjadas (Cephaleuros $s p$ ) fue mayor en fincas de marañón con manejo orgánico que en las fincas con manejo convencional.

La menor área bajo la curva de progreso de manchas necróticas (Pestalotia $s p$ ) se presentó en fincas de marañón con manejo orgánico que en las fincas con manejo convencional.

La menor área bajo la curva de progreso de manchas anaranjadas (Cephaleuros sp) se presentó en fincas de marañón con manejo convencional que en las fincas con manejo orgánico.

\section{REFERENCIAS BIBLIOGRÁFICAS}

Barnett, HL; Hunter, BB.1998. Illustrate Genera of Fungi. Fourth Ed.Minnesota.APS PRESS The American PhytopathologicalSociety. $218 \mathrm{p}$.

Castaño, ZJ. 1994. Guía para el diagnóstico de enfermedades en cultivos de importancia económica. Escuela Agrícola Panamericana. El Zamorano, Tegucigalpa, Honduras. 290 p.

CIPRES (Centro Parea la promoción, la investigación y el desarrollo rural y social). 2008. Guía para el manejo de las principales plagas de marañón orgánico en Nicaragua, 1era edición. 39 p.

Galdánez, A. 2004. Ministerio de Agricultura (MAG). Guía técnica del cultivo del marañón en el Salvador. Programa nacional de frutas de El Salvador, 1era Edición. 69 p.

INETER (Instituto Nicaragüense de Estudios Territoriales, NI). 2009. Dirección general de Meteorología. Resumen meteorológico diario del 2009 y 2010 . Managua, Nicaragua.

Muñoz, LC; Pérez, FV; Cobos, SP; Hernández, AR; Sánchez, PG. 2007. Sanidad Forestal: Guía en imágenes de plagas, enfermedades y otros agentes presentes en los bosques. 2da. Ed. Madrid, Ediciones Mundi-Prensa. 575 p.

Monterrosa, D. 1996. Técnicas fitopatológicas de laboratorio para el diagnóstico de las enfermedades de las plantas .Proyecto CATIEINTA-MIP (NORAD). Managua, Nicaragua. 29 p.

Ploestz, RC. 1998. Compendium of tropical fruitDiseases.Universidad de Florida. The American PhotopathologicalSociety (APS). 88 p.

Sánchez, M. 1988. Plagas y enfermedades de los frutales. 1ra. Ed. Cuba.Pueblo y educación. P 195.

SAS Institute, 2003. University of Nebraska. Cary, NC, USA.V.91.

Schard, D. 1988. Laboratory guide for identification of plants pathogenic Bacterial, 2a ed.Printed in the united State of America, by the American Phytopathology society.Minesota.EEUU.164 p.

Vanderplank, JE. 1963. Plant diseases: epidemiology and control. New York. Academia press. 69 p. 